\title{
Threshold corrections to inclusive jet production at hadron colliders
}

\section{M.C. Kumar*}

II. Institut für Theoretische Physik, Universität Hamburg

Luruper Chaussee 149, D-22761 Hamburg, Germany

E-mail: kumar.meduriedesy.de

\section{S. Moch ${ }^{a, b}$}

${ }^{a}$ II. Institut für Theoretische Physik, Universität Hamburg

Luruper Chaussee 149, D-22761 Hamburg, Germany

${ }^{b}$ Deutsches Elektronen-Synchrotron DESY, Platanenallee 6, D-15738 Zeuthen, Germany

E-mail: sven-olaf.mochedesy.de

We compute QCD threshold corrections to the inclusive jet hadro-production process in the softgluon resummation formalism. We expand the resummed results to 1-loop and 2-loop level at next-to-leading logarithmic (NLL) accuracy and compare with the results in the literature. Further, by presenting the transverse momentum distributions of the jet for Tevatron and LHC, we investigate the jet cone size dependence and the kinematical range of validity of these threshold corrections.

11th International Symposium on Radiative Corrections (Applications of Quantum Field Theory to Phenomenology) (RADCOR 2013),

22-27 September 2013

Lumley Castle Hotel, Durham, UK

\footnotetext{
* Speaker.
} 
The hadro-production of inclusive jet process is given by

$$
P\left(p_{A}\right)+P(\bar{P})\left(p_{B}\right) \rightarrow J\left(p_{J}\right)+X\left(p_{X}\right),
$$

where $p_{A}, p_{B}$ are the incoming hadron momenta, $p_{J}$ is the momentum of the observed jet $J$ and $p_{X}$ is the momentum of the inclusive final state $X$. This is a dominant process in hadron collisions and plays an important role in probing the hard scattering of partons at high energies. It has been very well measured experimentally both at the LHC and at the Tevatron [1-4], and the relevant data has been used in the new Physics searches as well as in the measurement of strong coupling constant $\alpha_{s}$ and gluon distribution functions inside the proton.

All such analyses require a very precise theoretical prediction of the jet cross sections, in particular the corresponding higher order QCD corrections. While the exact next-to-leading order (NLO) results to the parton scattering processes underlying the one-jet inclusive hadro-production are available since long $[5,6]$, the complete next-to-next-to-leading order (NNLO) cross sections are yet to be computed. The jet cross sections in the large transverse momentum $p_{T}$ or the threshold region are essentially dominated by the soft gluon contributions. In this region, the threshold logarithms are very large and need to be resummed to all orders in the perturbation theory. Such threshold corrections to 2-loop level at NLL accuracy have been computed in [7] and the all order resummation to NLL accuracy is done in [8,9]. Recently, the NNLO QCD corrections in the purely gluonic channel to one-jet inclusive and di-jet production at hadron colliders have been performed [10].

The jet cross sections precisely depend on the details of the jet definition i.e., the jet algorithm, the jet's cone size $R$ and the jet's mass. The resummation of threshold logarithms in [7] assumes massless jets while the resummed results given in [9] are obtained using small cone approximation where the jets are assumed to be massive. For the former case of massless jets, as the threshold corrections are widely used e.g., in experimental analysis of one-jet inclusive data [11] and in the determination of parton distribution functions (PDFs) from global fits [12,13], it is particularly interesting to assess their kinematical range of validity. To this end, in the present work we compute the threshold logarithms in the soft-gluon resummation formalism $[14,15]$ and compare our results at NLL accuracy [16] with those in the literature [7]. Further, to investigate their kinematical range of validity and their cone size dependence, we compare the 1-loop threshold corrections with the exact NLO QCD results. The latter can directly be obtained from stand-alone programs like NLOJET++ [17] and MEKS [18]. We find that threshold corrections provide good theory predictions but in a kinematical region that is rather limited to large $p_{T}$ and small jet cone sizes $R$. Since the latter turn out to be typically much smaller than the currently chosen values at LHC and Tevatron, the dependence on finite cone sizes, which is unaccounted for in [7], introduces a large additional systematic uncertainty in the threshold approximation. This is unlike the case of soft-gluon resummation for single-particle inclusive hadro-production at high transverse momentum [19] or for heavy-quark hadro-production (see, e.g., [20,21]), where the threshold logarithms are found to provide extremely precise predictions through NNLO.

There are 9 different subprocesses that contribute to this inclusive jet production and a generic subprocess can be written as

$$
a\left(p_{1}\right)+b\left(p_{2}\right) \rightarrow c\left(p_{3}\right)+d\left(p_{4}\right),
$$


where $a, b$ are the incoming partons and $c, d$ are the partons in the final state. The corresponding Mandelstam invariants are $s=\left(p_{1}+p_{2}\right)^{2}, t=\left(p_{1}-p_{3}\right)^{2}$ and $u=\left(p_{2}-p_{3}\right)^{2}$. Here, either of the partons in the final state can form a jet (the other being inclusive) and hence the cross sections can be computed either by symmetrizing the matrix elements $(t \leftrightarrow u)$ or by running a jet-algorithm. Defining $s_{4}=s+t+u \geq 0$, the threshold region can be identified as $s_{4}=0$. In a physical interpretation $s_{4}$ denotes the additional energy carried away by the real gluons above the partonic threshold.

For one-particle inclusive (1PI) kinematics [22], the threshold corrections at a certain order can be obtained from the expansion of the resummed result to that particular order. The resummation is based on the factorization of the partonic cross sections near threshold into various functions, e.g. soft, hard and jet functions. The soft function $S$ is governed by the soft anomalous dimension $\Gamma_{S}[8,23]$ and it summarizes the dynamics of soft gluons that are not collinear to the external partons. The hard scattering of the incoming partons is described by the hard function $H$ and the dynamics of both initial and final state collinear gluons are summarized respectively by the initial and final state jet functions $\mathcal{I}^{I}$ and $g^{F}$ which contain all leading logarithms LL and some NLL enhancements.

For threshold corrections, it is precisely these final state jet functions $\mathcal{I}_{b}^{F}$ that include the $R$ dependence. For massless jets, these jet functions will be independent of $R$ as in [7]. Any dependence of the final state jet functions on $R$ will alter the resummed cross sections at LL accuracy and instead give rise to $\ln R$ terms leading to jet's mass, as is the case for [9].

The resummation is conveniently carried out in the space of moments $N$. The details of the process dependent soft, hard and soft anomalous dimensions together with the jet functions for massless jets and the parton level resummed formula for a generic subprocess in Eq. (2) can be found in $[7,16,23]$. Moreover, these resummed results can be expanded to obtain the threshold corrections which for a generic subprocess to 1-loop level at NLL accuracy can be given as

$$
\left.s^{2} \frac{d^{2} \hat{\sigma}}{d t d u}=\frac{\alpha_{s}}{\pi} \sigma^{(0)}\left\{c_{2}\left[\frac{\ln \left(s_{4} / p_{T}^{2}\right.}{s_{4}}\right)\right]_{+}+c_{1}\left[\frac{1}{s_{4}}\right]_{+}\right\},
$$

where $\sigma^{(0)}$ is the Born cross section. The corresponding 2-loop level threshold corrections are given by

$$
s^{2} \frac{d^{2} \hat{\sigma}}{d t d u}=\left(\frac{\alpha_{s}}{\pi}\right)^{2} \sigma^{(0)}\left\{b_{2}\left[\frac{\ln ^{3}\left(s_{4} / p_{T}^{2}\right)}{s_{4}}\right]_{+}+b_{1}\left[\frac{\ln ^{2}\left(s_{4} / p_{T}^{2}\right)}{s_{4}}\right]_{+}\right\} .
$$

Here $c_{i}\left(b_{i}\right)$ are the coefficients of $\operatorname{LL}(i=2)$ and $\operatorname{NLL}(i=1)$ at 1-loop (2-loop) level. The subleading logarithms following NLL contain the hard matching functions that can be obtained from the finite parts of the 1-loop virtual graphs. In the present analysis, though, we have not included these matching functions and leave them for future study. The necessary formulae for these matching functions in various kinematics have been derived in [19,24].

In our analytical computation we have used the symbolic manipulation program FORM [25] and the related color package [26] for color algebra. A complete treatment of the kinematics and phase space integration can be found in [27] and the plus-distributions are defined as in [22]. We find that our analytical results for all parton level cross sections are in good agreement with those given in [7] except for a small difference of an overall color factor of $\left[N_{c}^{2} /\left(N_{c}^{2}-1\right)^{2}\right]$ at NLL level for the 
subprocess $g g \rightarrow q \bar{q}$. The relative contribution of this subprocess to the total cross section is very small for both Tevatron and LHC energies, hence the differences are numerically negligible in any application for collider phenomenology.

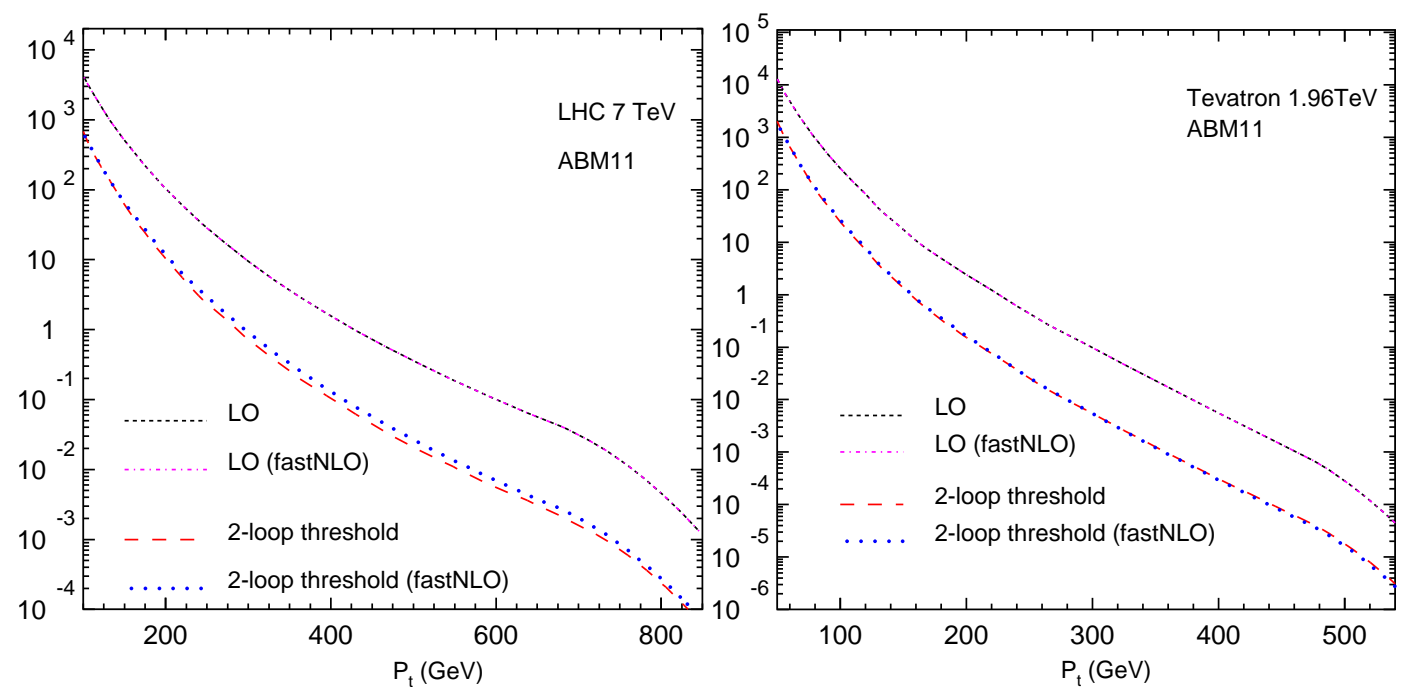

Figure 1: LO results and 2-loop threshold corrections $\sigma^{(2)}$ for the transverse momentum distribution of the jet at the LHC (left) and at the Tevatron (right).

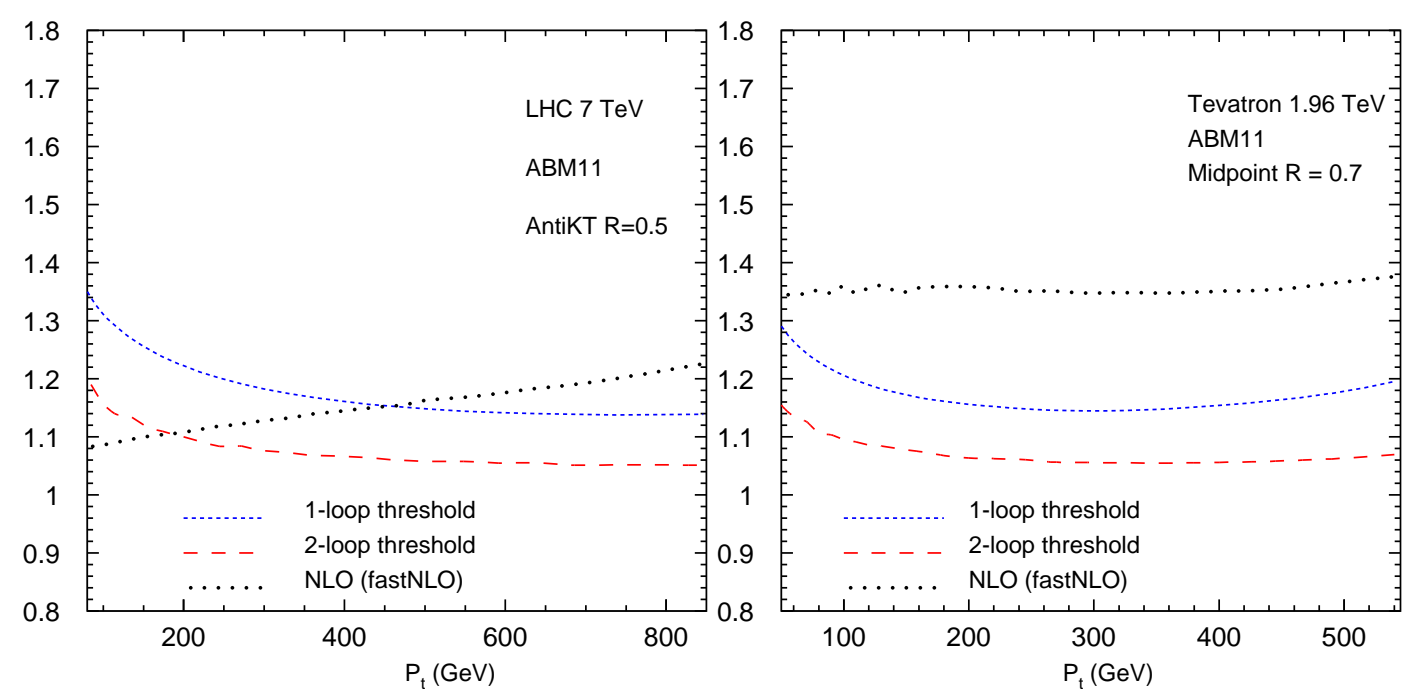

Figure 2: $K$-factors $K^{(1)}, K^{(2)}$ and $K^{(N L O)}$ defined with respect to 1-loop threshold corrections, 2-loop threshold corrections and the exact NLO results for $\sqrt{S}=7 \mathrm{TeV}$ LHC (left) and for Tevatron (right).

Next we present the transverse momentum distributions of the jet for both Tevatron $(\sqrt{S}=1.96$ $\mathrm{TeV})$ and LHC $(\sqrt{S}=7 \mathrm{TeV})$ energies, and compare our threshold corrections with those obtained from fastNLO [28]. In our analysis, we use CTEQ6.6 $\left(\alpha_{s}\left(M_{Z}^{2}\right)=0.118\right)$ [29] and ABM11 NNLO $\left(\alpha_{s}\left(M_{Z}^{2}\right)=0.1134\right)$ [12] PDFs. Here $\alpha_{s}$ is provided by the respective PDF sets through the LHAPDF interface [30]. Throughout our analysis, we use the scale choice $\mu_{F}=\mu_{R}=p_{T}$. For our numerical study, we consider the central rapidity region of the jet, $0 \leq|y| \leq 0.5$ for LHC and $0 \leq y \leq 0.4$ for Tevatron, where the parton momentum fractions $x_{1}$ and $x_{2}$ are closer to each other. In the rest of 


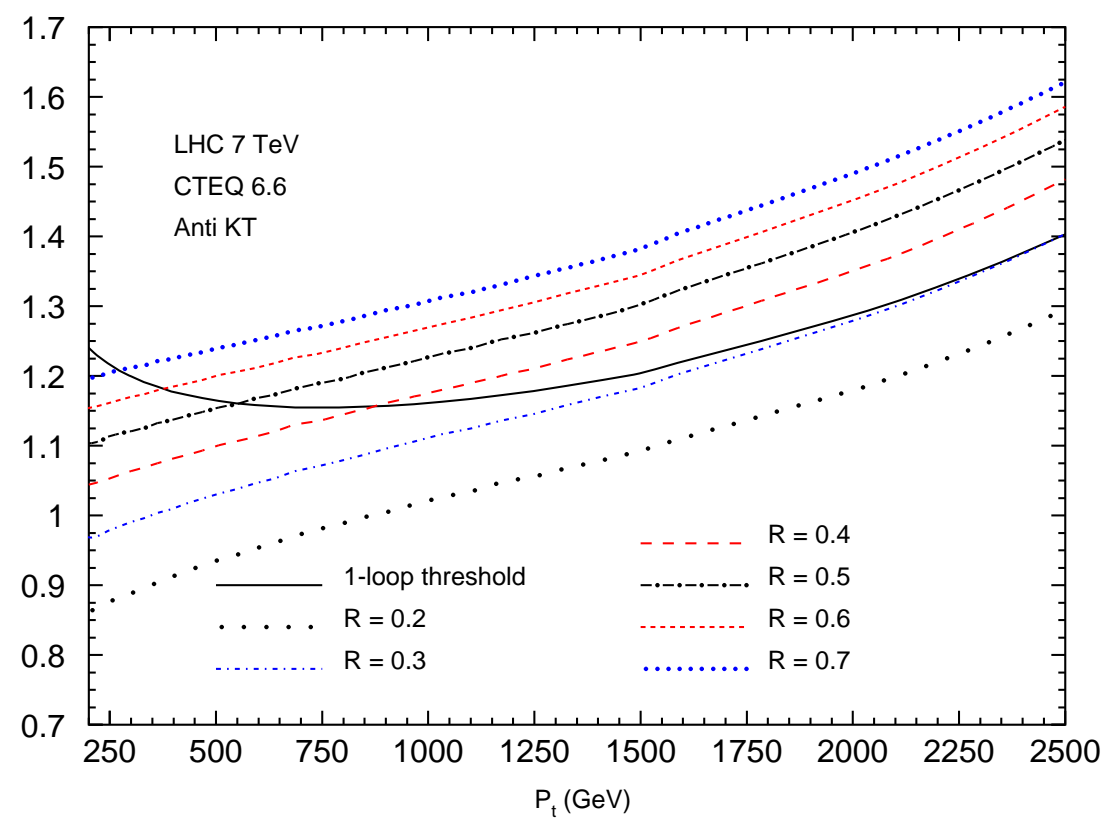

Figure 3: NLO $K$-factors $K^{(N L O)}$ for inclusive jet production as a function of the parameter $R$ in the anti- $k_{t}$ jet algorithm, computed for $\sqrt{S}=7 \mathrm{TeV}$ LHC. The solid line corresponds to the one-loop threshold corrections $K^{(1)}$ at NLL accuracy.

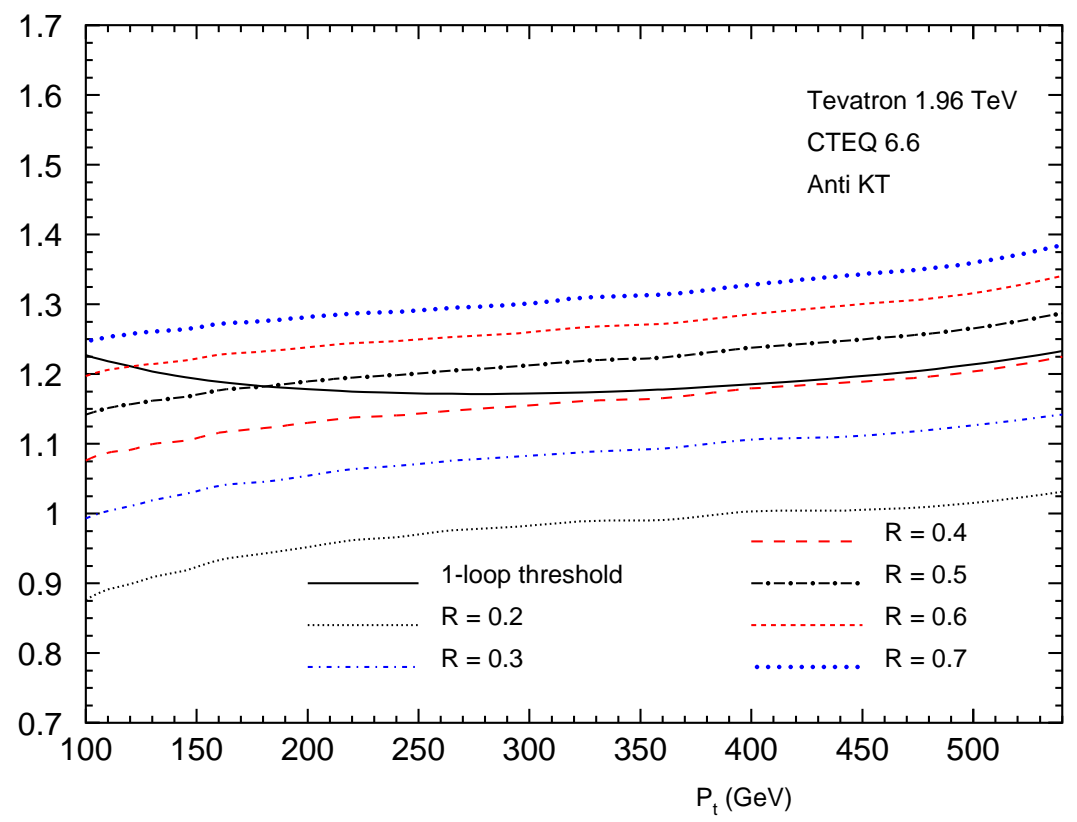

Figure 4: NLO $K$-factors $K^{(N L O)}$ for inclusive jet production as a function of the parameter $R$ in the anti- $k_{t}$ jet algorithm, computed for Tevatron $\sqrt{S}=1.96 \mathrm{TeV}$. The solid line corresponds to the one-loop threshold corrections $K^{(1)}$ at NLL accuracy. 
the paper we use the following $K$-factors defined as:

$$
\begin{gathered}
K^{(1)}=1+\frac{\sigma^{(1)}}{\sigma^{(0)}}, \quad K^{(2)}=1+\frac{\sigma^{(2)}}{\sigma^{(0)}}, \\
K^{(N L O)}=1+\frac{\sigma^{(N L O)}}{\sigma^{(0)}}, \quad K^{(N N L O *)}=1+\frac{\sigma^{(N L O)}+\sigma^{(2)}}{\sigma^{(0)}},
\end{gathered}
$$

where $\sigma^{(0)}$ is the LO cross section, $\sigma^{(1)}$ and $\sigma^{(2)}$ are the 1-loop and 2-loop threshold corrections to NLL accuracy, and $\sigma^{(N L O)}$ is the exact NLO QCD correction.

In Fig. (11), we show the comparison of the LO cross sections and the 2-loop threshold corrections $\sigma^{(2)}$ for LHC as well as Tevatron, and find that our results are well in agreement with those of fastNLO. For the 2-loop threshold corrections $\sigma^{(2)}$ this constitutes an independent check of [7]. Next, we validate the threshold corrections by comparing them with the fixed order NLO QCD results. In Fig. (2), we present the $K$-factors $K^{(1)}, K^{(2)}$ and $K^{(N L O)}$. The NLO results for $K^{(N L O)}$ are read from the grids of fastNLO. For LHC, the NLO results are obtained using the anti- $k_{t}$ jet algorithm $(R=0.5)$ and are used in the CMS inclusive jet data analysis [2], whereas for Tevatron they are computed using the mid-point cone algorithm $(R=0.7)$.

In the high $p_{T}$ region or the threshold region $\left(s_{4}=0\right)$, the Sudakov logarithms dominate in the perturbative expansion and hence the 1-loop threshold corrections are expected to be closer to the NLO QCD corrections, i.e., $K^{(1)} \simeq K^{(N L O)}$. But as can be seen from Fig. (2), these two predictions differ for most of the $p_{T}$ region considered. For LHC, in the low $p_{T}$ region the threshold corrections overestimate the NLO ones. Particularly the 2-loop threshold corrections are larger than even the NLO ones and are subject to large theory uncertainties in the relevant jet analysis. The source of discrepancy can be related to the assumption of massless jets and the missing hard matching functions in the computation of threshold corrections.

To investigate this further, one needs to consider the jet definition used in the exact NLO results, in particular the parameter $R$. At LO the two partons in the final state, which eventually hadronize and form two jets, are well separated in the rapidity-azimuthal angular plane and hence LO results are insensitive to the choice of $R$. However, at NLO and beyond there are additional partons in the final state and their formation into jets very much depends on the size of the cone in which these partons fall. This is completely different from the case of resummation of massless jets where there is no dependence on $R$. The deviations of $K^{(N L O)}$ from $K^{(1)}$ can better be understood from the variation of NLO results with $R$. For this variation, we use the NLOJET++ program with the anti- $k_{t}$ jet algorithm from FastJet [31], and CTEQ6.6 PDFs [29]. It is worth noting that these NLO cross sections can also be computed using the results of "small-cone approximation" (SCA) [32] that are found to be applicable up to cone sizes of $R=0.7$.

In Figs. (3) and ( (4), we present our results in terms of $K^{(N L O)}$ for LHC and Tevatron by varying the cone size from $R=0.2$ to $R=0.7$. These figures demonstrate that the NLO QCD corrections increase with $R$ and the uncertainty in the NLO results due to this cone size variation can be as large as $30 \%$ regardless of the $p_{T}$ range considered. Interestingly, in the high $p_{T}$ region the threshold approximation which is independent of $R$ coincides with the exact NLO results for smaller $R$ values of about $R=0.3$. It is also worth noting here that the 2-loop threshold corrections for the Tevatron illustrated in Fig. (1) have been used in the determination of $\alpha_{s}[11]$ and they have decreased the 


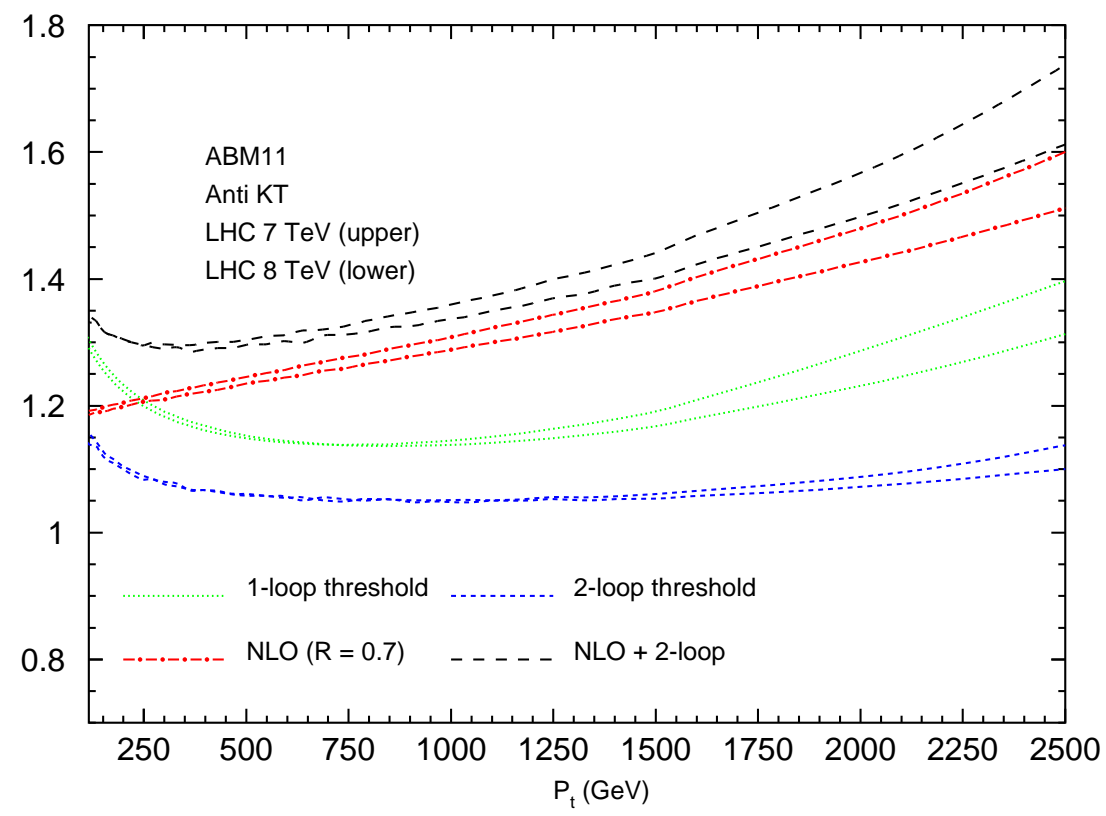

Figure 5: Comparison of $K$-factors $K^{(1)}, K^{(2)}, K^{(N L O)}$ and $K^{(N N L O *)}$ for 1-loop threshold, 2-loop threshold, NLO and NLO + 2-loop (NNLO*) cross sections computed for $\sqrt{S}=7,8 \mathrm{TeV}$ LHC.

central value of $\alpha_{s}\left(M_{Z}^{2}\right)=0.1201$ obtained from pure NLO QCD corrections to $\alpha_{S}\left(M_{Z}^{2}\right)=0.1161$. Finally, in Fig. (5), we present the $K$-factors $K^{(1)}, K^{(2)}, K^{(N L O)}$ and $K^{(N N L O *)}$ for $\sqrt{S}=7,8 \mathrm{TeV}$ LHC, for a chosen $R=0.7$.

To improve the description of 1-loop threshold corrections in the low $p_{T}$ region $(<500 \mathrm{GeV})$, where $K^{(1)}$ is larger than $K^{(N L O)}$, one requires to systematically include the hard matching functions. We also note that in a recent study [33], which is closely related to the formalism of "one particle inclusive" (1PI) kinematics that we are considering, the authors corroborate our findings and, using the "narrow jet approximation" they systematically include the dependence of the jet cone size $R$ in the threshold corrections.

To summarize, we have computed the threshold corrections to inclusive jet production at hadron colliders in the soft-gluon resummation formalism and find that our results are in agreement with those in the literature apart from few typographical errors. Furthermore, we have investigated their kinematical range of validity by studying 1-loop threshold and exact NLO QCD results. These QCD threshold corrections provide better approximation in the high $p_{T}$ region and for smaller cone sizes of about $R=0.3$, implying that these threshold corrections can lead to large theory uncertainties for low $p_{T}<500$ values and for typical values of $R$ used at LHC or Tevatron. 


\section{References}

[1] ATLAS Collaboration, G. Aad et al., Phys.Rev. D86, 014022 (2012), arXiv:1112.6297.

[2] CMS Collaboration, S. Chatrchyan et al., Phys.Rev.Lett. 107, 132001 (2011), arXiv:1106.0208.

[3] CDF Collaboration, T. Aaltonen et al., Phys.Rev. D78, 052006 (2008), arXiv:0807.2204.

[4] D0 Collaboration, V. Abazov et al., Phys.Rev.Lett. 101, 062001 (2008), arXiv:0802.2400.

[5] S. D. Ellis, Z. Kunszt, and D. E. Soper, Phys.Rev.Lett. 64, 2121 (1990).

[6] W. Giele, E. N. Glover, and D. A. Kosower, Phys.Rev.Lett. 73, 2019 (1994), arXiv:hep-ph/9403347.

[7] N. Kidonakis and J. Owens, Phys.Rev. D63, 054019 (2001), arXiv:hep-ph/0007268.

[8] N. Kidonakis, G. Oderda, and G. F. Sterman, Nucl.Phys. B525, 299 (1998), arXiv:hep-ph/9801268.

[9] D. de Florian and W. Vogelsang, Phys.Rev. D76, 074031 (2007), arXiv:0704.1677.

[10] A. G.-D. Ridder, T. Gehrmann, E. Glover, and J. Pires, (2013), arXiv:1301.7310.

[11] D0 Collaboration, V. Abazov et al., Phys.Rev. D80, 111107 (2009), arXiv:0911.2710.

[12] S. Alekhin, J. Blümlein, and S. Moch, Phys.Rev. D86, 054009 (2012), arXiv:1202.2281.

[13] A. Martin, W. Stirling, R. Thorne, and G. Watt, Eur.Phys.J. C63, 189 (2009), arXiv:0901.0002.

[14] H. Contopanagos, E. Laenen, and G. Sterman, Nucl. Phys. B484, 303 (1997), hep-ph/9604313.

[15] S. Catani, M. L. Mangano, P. Nason, and L. Trentadue, Nucl. Phys. B478, 273 (1996), hep-ph/9604351.

[16] M. C. Kumar and S. Moch, (2013), arXiv:1309.5311.

[17] Z. Nagy, Phys. Rev. Lett. 88, 122003 (2002), hep-ph/0110315.

[18] J. Gao et al., Comput.Phys.Commun. 184, 1626 (2013), arXiv:1207.0513.

[19] S. Catani, M. Grazzini, and A. Torre, Nucl.Phys. B874, 720 (2013), arXiv:1305.3870.

[20] R. Bonciani, S. Catani, M. L. Mangano, and P. Nason, Nucl. Phys. B529, 424 (1998), hep-ph/9801375.

[21] S. Moch, P. Uwer, and A. Vogt, Phys.Lett. B714, 48 (2012), arXiv:1203.6282.

[22] E. Laenen, G. Oderda, and G. F. Sterman, Phys.Lett. B438, 173 (1998), arXiv:hep-ph/9806467.

[23] N. Kidonakis, G. Oderda, and G. F. Sterman, Nucl.Phys. B531, 365 (1998), arXiv:hep-ph/9803241.

[24] R. Kelley and M. D. Schwartz, Phys.Rev. D83, 045022 (2011), arXiv:1008.2759.

[25] J. Vermaseren, (2000), arXiv:math-ph/0010025.

[26] T. van Ritbergen, A. Schellekens, and J. Vermaseren, Int.J.Mod.Phys. A14, 41 (1999), arXiv:hep-ph/9802376.

[27] W. Beenakker, H. Kuijf, W. van Neerven, and J. Smith, Phys.Rev. D40, 54 (1989).

[28] M. Wobisch, D. Britzger, T. Kluge, K. Rabbertz, and F. Stober, (2011), arXiv:1109.1310.

[29] P. M. Nadolsky et al., Phys.Rev. D78, 013004 (2008), arXiv:0802.0007.

[30] M. Whalley, D. Bourilkov, and R. Group, (2005), arXiv:hep-ph/0508110.

[31] M. Cacciari, G. P. Salam, and G. Soyez, Eur.Phys.J. C72, 1896 (2012), arXiv:1111.6097.

[32] B. Jager, M. Stratmann, and W. Vogelsang, Phys.Rev. D70, 034010 (2004), arXiv:hep-ph/0404057.

[33] D. de Florian, P. Hinderer, A. Mukherjee, F. Ringer, and W. Vogelsang, (2013), arXiv:1310.7192. 